\title{
Assessment of torque angle of brackets from different brands
}

\author{
Anelisa dos Anjos ${ }^{1}$, Daniela Daufenback Pompeo², Gilson José Enricone dos Anjos ${ }^{1}$, \\ Gustavo Mussi Stefan Oliveira ${ }^{3}$, Henrique Damian Rosário ${ }^{1}$
}

\author{
${ }^{1}$ Faculdades Integradas do Norte de Minas - FUNORTE, Dental School, Department of Orthodontics, Florianópolis, SC, Brazil \\ ${ }^{2}$ Universidade Sagrado Coração - USC, Dental School, Department of Oral Biology, Bauru, SP, Brazil \\ ${ }^{3}$ University of Louisville School of Dentistry, Department of General Dentistry \& Oral Medicine, Louisville, KY, USA
}

Received for publication: December 15, 2014 Accepted: March 20, 2015

Correspondence to: Henrique Damian Rosário Rua Adhemar da Silva, 235, Sala 3, Kobrasol CEP: 88101-090 - São José, SC, Brasil Phone: +55 4830351211 Fax: +55 4830351211 E-mail: drhenriquerosario@icloud.com

\begin{abstract}
Aim: To measure torque angle values of brackets designed for canines, comparing it to their prescription values. Methods: One hundred and sixty maxillary $\left(-2^{\circ}\right)$ and mandibular $\left(-11^{\circ}\right)$ canine brackets of Roth prescription from the following brands were selected: Abzil, Eurodonto, Morelli and Ormco. The brackets were set in wax and images were obtained by scanning. Reference points were determined over these images, lines drawn and the torque angle was measured. The Student's t-test and the Wilcoxon signed-rank test were used at a significance level of $5 \%$. Results: For maxillary canine brackets, the medium torque angle values were: Abzil $0.93^{\circ}\left( \pm 0.88^{\circ}\right)$; Eurodonto $0.13^{\circ}\left( \pm 0.34^{\circ}\right)$; Morelli $-2.56^{\circ}\left( \pm 0.50^{\circ}\right)$, and Ormco $-1.16^{\circ}\left( \pm 1.27^{\circ}\right)$. For mandibular canine brackets, the values were: Abzil - $11.76^{\circ}\left( \pm 0.40^{\circ}\right)$; Eurodonto $-10.40^{\circ}( \pm$ $\left.0.25^{\circ}\right)$; Morelli $-11.18^{\circ}\left( \pm 0.56^{\circ}\right)$, and Ormco $-11.36^{\circ}\left( \pm 0.30^{\circ}\right)$. For maxillary canine brackets, the brands Abzil, Morelli, and Ormco presented statistically diferente values from those indicated for prescription. For mandibular canine brackets, the brands Abzil, Eurodonto, and Ormco presented statistically different values from the prescribed ones. Conclusions: Some marketed brands present differences between the torque angle found in the brackets and those recommended in the prescription. However, these differences are clinically acceptable.
\end{abstract}

Keywords: orthodontic brackets; torque; orthodontics.

\section{Introduction}

During the 70's, Andrews introduced the Straight-Wire appliance and the 11 Andrews' prescriptions. The concept described a completely programmed appliance, created by a tridimensional system of brackets. These brackets were designed with ideal angular characteristics of each tooth, for a regular occlusion, adjustments embedded in brackets and ideal tooth positioning ${ }^{1-3}$.

Another characteristic of these brackets is torque, which may be defined from clinical and mechanical standpoints ${ }^{4}$. Regarding mechanics, it indicates torsion of a structure in its longitudinal axis resulting in a torsion angle. Clinically, it represents a buccolingual inclination of the crown/root of a tooth. The terms moment, moment of torsion, biomechanics torque and third-order torque can also be found in the literature.

Roth $^{5}$ modified some of the bracket prescription values from the original Straight-Wire system, producing a universal prescription that is used in every case, and named it the second generation of pre-adjusted appliances. For maxillary canines, a decrease in lingual crown torque from $-7^{\circ}$ to $-2^{\circ}$ was recommended and 
for mandibular canines, the $-11^{\circ}$ lingual crown torque was maintained. A mesial rotation of $4^{\circ}$ in maxillary canines and $2^{\circ}$ in mandibular canines was also added.

Straight-wire appliances correct dental positions if brackets are well placed by the orthodontist, and dental crowns present a typical morphology ${ }^{6}$. The expression of torque over teeth is influenced by factors such as: anatomy of the buccal aspect of the tooth, tooth size, bracket position, bracket $\operatorname{size}^{7}$, gap between the wire and the bracket slot ${ }^{8-9}$, differences in direct bonding ${ }^{10}$, unusual crown shape and angulation $^{11}$, application of strength outside the tooth center of resistance ${ }^{5}$, dental arch shape and dental eruption position ${ }^{12}$.

Due to the number of possible variations in the torque expression of a bracket, it is important that their angulation values meet the values recommended by the author of the technique, so that the orthodontic treatment is successful, resulting in favorable occlusion, function and esthetic conditions.

The aim of this study was to assess the agreement among the torque angle values of metallic brackets in the Roth prescription from four different commercial brands, with what was recommended in the prescriptions.

\section{Material and methods}

This laboratory study used a total of 160 metallic orthodontic brackets of four commercial brands with $0.022 " \mathrm{x}$ 0.028 " slot of the Roth prescription. The sample was divided in 4 groups of brackets $(n=40$, with 20 maxillary canines and 20 mandibular canines), according to the respective commercial brands: Group 1 - 3M Abzil brackets (São José do Rio Preto, SP, Brazil); model Kirium Line; Group 2 - Eurodonto brackets (Curitiba, PR, Brazil); model 280; Group 3 - Morelli brackets (Sorocaba, SP, Brazil); model Roth Max; Group 4 - Ormco brackets (Glendora, CA, USA); model mini-Damon.

For image capturing and more accurate torque measurement, the bracket was placed over a glass plate, with the mesial aspect facing down (in contact with the glass plate surface). A plastic set square (Professional drawing set square, Acrimet, São Paulo, SP, Brazil) was used to place the bracket along with the utility wax (Wilson Polidental, Cotia, $\mathrm{SP}$, Brazil), measuring $1.5 \mathrm{~cm} \mathrm{x} 1.0 \mathrm{~cm} \mathrm{x} 0.5 \mathrm{~cm}$ (Figure 1). The wax stabilized the bracket so it could be scanned (Scanner Epson Stylus TX 220 ${ }^{\circledR}$, Taiwan, China) for image capturing, providing a total of 160 images.

The identification of brackets was performed during the scanning process, considering the first letter as the commercial brand, the two following numbers indicated the tooth, and the two subsequent ones indicated the order of image capturing. For example, a bracket with the specification "Morelli, tooth 13, number 1" would receive the following nomination: M130. Numeration was omitted during measurements so that the evaluator was blind to which group was being assessed.

The images were transferred to a Notebook computer (Acer Ink Aspire ${ }^{\circledR}$ 4740, I3, 3MB, 250GB, Taiwan, China). The resolution used was 9600 pixels for an area of $1.016 \mathrm{~cm}^{2}$.

Some points and lines of reference were drawn to measure

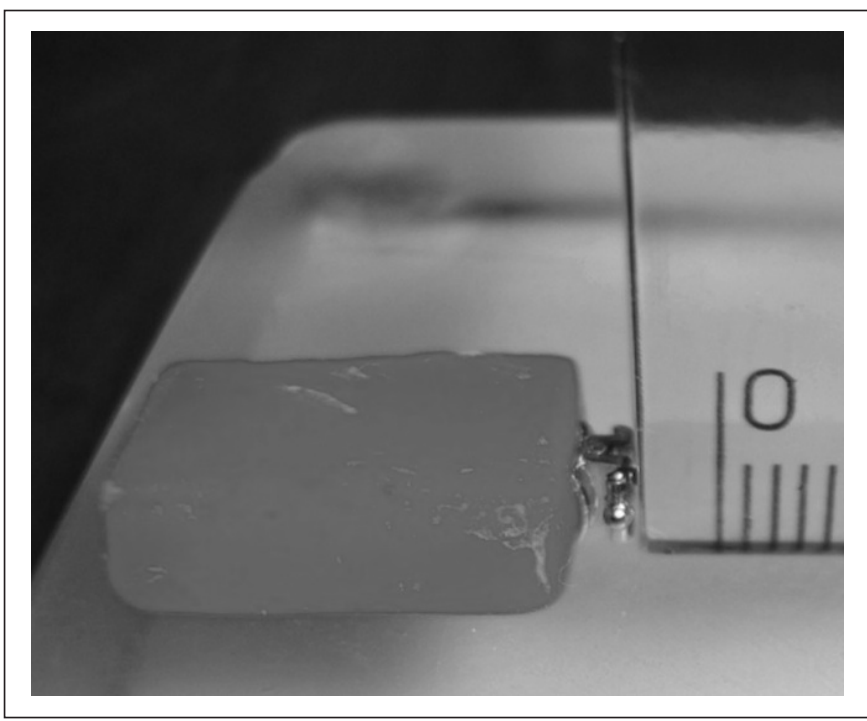

Fig. 1 - Bracket positioning over glass plate, stabilized in the wax block for later image capturing.

torque according to Streva ${ }^{13}$, and are shown in Figures 2 and 3. Point B1: Point of cervical edge of the bracket body base. Point B2: Point of occlusal edge of the bracket body base. Point C1: Angle vertex between the base of the bracket slot and the gingival flap.

Point C2: Angle vertex between the base of bracket slot and the incisal slot edge.

Line B: Resulting from the junction of B1 and B2 points, it defined the base of the bracket body.

Line C: Resulting from the junction of $\mathrm{C} 1$ and $\mathrm{C} 2$ points. This line defined the slot base, and therefore its torque.

The torque angle was defined by the angle formed between the bracket body base (line B), and the slot floor (line C), as illustrated in Figure 3. A single evaluator measured these angles during three consecutive days. For this purpose, the images were viewed on the Windows $7^{\circledR}$ display and software MB-Ruler ${ }^{\circledR}$ (Figure 4) was used. Then, the data were recorded in a spreadsheet.

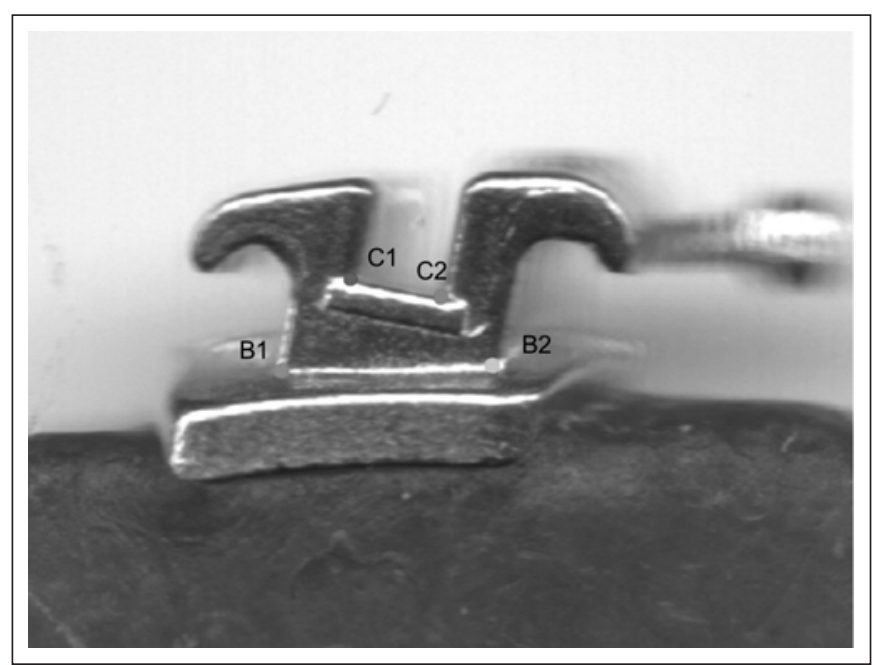

Fig. 2 - Points B1 and B2 in the base of the bracket body. Points C1 and C2 limiting the floor of the slot of the bracket. 


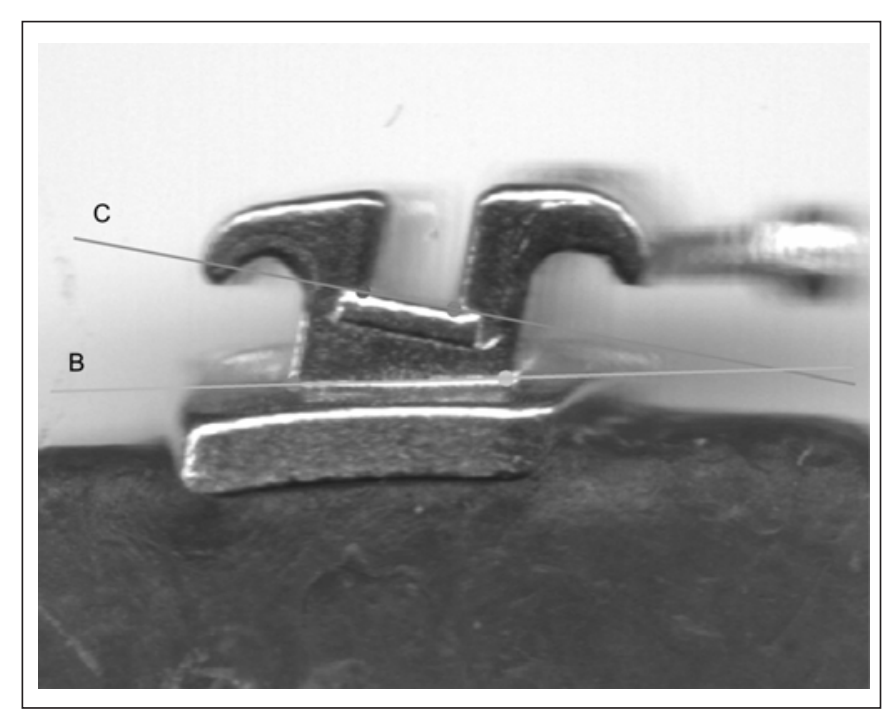

Fig. 3 - Reference lines used to measure torque angle. Line B, and line C.

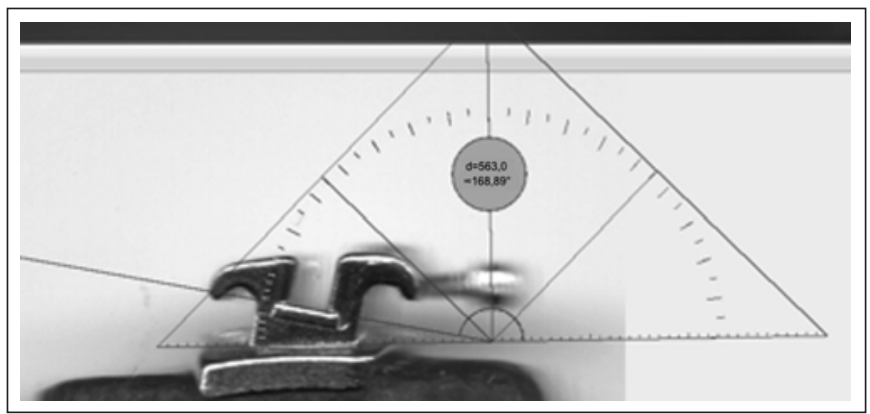

Fig. 4 - Application of software MB Ruler ${ }^{\circledR}$ to measure torque angle.

\section{Assessment of Measurement Error}

Two images of maxillary brackets and two images of mandibular brackets of each assessed brand were randomly selected for analysis of method error; the evaluator measured each image at least twice with a 7-day interval between measurements.

The paired t-test was used to verify the intra-examiner systematic error for both repetitions. Dahlberg error calculation was used to determine the casual error ${ }^{13}$ : Error $V / \Sigma \mathrm{d}^{2} / 2 \mathrm{n}$ considering $\mathrm{d}=$ difference between $1^{\text {st }}$ and $2^{\text {nd }}$ measurements, and $n=$ number of measurements replications.

The results of the systematic error assessment and the casual error are shown in Table 1 . The method error estimate was between $0.18^{\circ}$ and $0.25^{\circ}$.

\section{Data Analysis}

Data were described by mean, standard deviation, minimum value, and maximum value parameters. The Shapiro-Wilk test was used to verify whether the groups were regularly distributed. For groups 1,2 and 4, the maxillary canine brackets were not regularly distributed. The mandibular brackets of groups 1,2 and 4 were regularly distributed. In group 3, all brackets (maxillary and lower mandibular) were regularly distributed.

The Student's t-test was used for groups with regular distribution to compare the values obtained with the norm, and the Wilcoxon signed-rank test was used in groups with irregular distribution. One-criterion variance analysis and the Tukey's post hoc test were used to compare brands, the difference among the obtained values and the norm in groups of mandibular canine brackets. The Kruskal-Wallis nonparametric test and the Miller's post hoc test were used in groups of maxillary canine brackets. A significance level of $5 \%(\mathrm{p}<0.05)$ was set for all statistical tests.

\section{Results}

The values of medium, minimum and maximum torque angle, and standard deviations of maxillary canine brackets are described in Table 2. The torque values of Eurodonto brackets were not statistically different when compared with the recommended norm.

Table 3 presents the results obtained for mandibular canine brackets. Mandibular brackets of the Morelli brand presented torque values that were not statistically different from the norm. The remaining brands presented statistically significant differences. The comparison among all four brands' values and the norm, in maxillary and mandibular canine brackets, is described in Tables 4 and 5, respectively.

\section{Discussion}

Streva et al. ${ }^{14}$ and Bóbbo ${ }^{15}$ studied the values of bracket torque angles, and compared them with the values recommended by certain techniques. They set the brackets in a jig, capturing images by light microscopy. The present study used a methodology that allowed the correct placement of the bracket, since the use of a set square perpendicular to the bracket would not allow its rotation, which could interfere in the image recording and torque angle measuring processes. Image was obtained by scanning.

Table 1 - Mean, standard deviation of both measurements, paired t-test, and Dahlberg error to assess the systematic error and casual error

\begin{tabular}{llllllll}
\hline Comparison & \multicolumn{2}{c}{ Measurement 1 } & \multicolumn{2}{l}{ Measurement 2 } & T & p & Error \\
& Mean & Sd & Mean & Sd & & & \\
$1^{\text {st }} \times 2^{\text {nd }}$ & -6.26 & 5.38 & -6.29 & 5.48 & 0.432 & $0.672 \mathrm{~ns}$ & 0.18 \\
$1^{\text {st }} \times 3^{\text {rd }}$ & -6.26 & 5.38 & -6.30 & 5.44 & 0.433 & $0.671 \mathrm{~ns}$ & 0.25 \\
$2^{\text {nd }} \times 3^{\text {rd }}$ & -6.29 & 5.48 & -6.30 & 5.44 & 0.110 & $0.914 \mathrm{~ns}$ & 0.25 \\
\hline
\end{tabular}

ns - non-significant statistical difference. 
Table 2 - Mean, standard deviation, minimum, maximum, and comparison to the norm of maxillary canine brackets.

\begin{tabular}{|c|c|c|c|c|c|c|c|}
\hline Brand & Mean & $S d$ & $\min$. & $\max$. & norm & differ. & $p$ \\
\hline Abzil & -0.93 & 0.88 & -2.16 & 0.00 & -2 & 1.07 & $0.001^{*}$ \\
\hline Eurodonto & -0.13 & 0.34 & -1.39 & 0.14 & 0 & -0.13 & $0.060 \mathrm{~ns}$ \\
\hline Morelli & -2.56 & 0.50 & -3.40 & -1.72 & -2 & -0.56 & $<0.001^{*}$ \\
\hline Ormco & -1.16 & 1.27 & -2.76 & 1.84 & 0 & -1.16 & $0.008^{*}$ \\
\hline
\end{tabular}

* - significant statistical difference $(p<0.05)$.

ns - non-significant statistical difference.

Table 3 - Mean, standard deviation, minimum, maximum, and comparison to the norm of mandibular canine brackets.

\begin{tabular}{|c|c|c|c|c|c|c|c|}
\hline Brand & Mean & Sd & $\min$. & $\max$. & Norm & differ. & $p$ \\
\hline Abzil & -11.76 & 0.40 & -12.53 & -11.09 & -11 & -0.76 & $<0.001^{*}$ \\
\hline Eurodonto & -10.40 & 0.25 & -10.88 & -10.04 & -11 & 0.60 & $<0.001^{*}$ \\
\hline Morelli & -11.18 & 0.56 & -12.29 & -10.28 & -11 & -0.18 & $0.173 \mathrm{~ns}$ \\
\hline Ormco & -11.36 & 0.30 & -11.90 & -11.00 & -11 & -0.36 & $<0.001^{*}$ \\
\hline
\end{tabular}

* - significant statistical difference $(p<0.05)$

ns - non-significant statistical difference.

Table 4 - Comparison among all four brands of value differences obtained, and the norm from each manufacturer in maxillary canine brackets.

\begin{tabular}{lllllll}
\hline Brand & Differ. & Sd & min. & $\max$. & $p$ & \\
Abzil & 1.07 & 0.88 & -0.16 & 2.00 & $<0.001^{*}$ & $\mathrm{~A}$ \\
Eurodonto & -0.13 & 0.34 & -1.39 & 0.14 & $\mathrm{~B}$ \\
Morelli & -0.56 & 0.50 & -1.40 & 0.28 & $\mathrm{BC}$ \\
Ormco & -1.16 & 1.27 & -2.76 & 1.84 & $\mathrm{C}$ \\
\hline
\end{tabular}

* - significant statistical difference $(p<0.05)$.

Brands with the same letter do not present significant statistical difference between themselves.

Table 5 - Comparison among all four brands of value differences obtained, and the norm from each manufacturer in mandibular canine brackets

\begin{tabular}{lllllll}
\hline Brand & Differ. & sd & min. & $\max$. & $\mathbf{p}$ & \\
Abzil & -0.76 & 0.40 & -1.53 & -0.09 & $<0.001^{*}$ & $\mathrm{~A}$ \\
Eurodonto & 0.60 & 0.25 & 0.12 & 0.96 & & $\mathrm{~B}$ \\
Morelli & -0.18 & 0.56 & -1.29 & 0.72 & & $\mathrm{Ab}$ \\
Ormco & -0.36 & 0.30 & -0.90 & 0.00 & & $\mathrm{Ab}$
\end{tabular}

* - significant statistical difference $(p<0.05)$

Brands with the same letter do not present significant statistical difference among themselves.

The reference points used for measurement were the same established in the works by Streva et al. ${ }^{14}$ and Bóbbo ${ }^{15}$, which made torque angles easy to identify. Using these points also allowed the results to offer a standard of comparison to those studies.

Bóbbo $^{15}$ analyzed the torque of maxillary and mandibular incisor brackets, and Streva et al..$^{14}$ assessed maxillary and mandibular canine brackets. Both assessed the brackets of the M.B.T. prescription from different commercial brands. Generally, the researchers observed that some brands obtained statistically different torque angle values from what was recommended by the M.B.T. prescription. The results observed in this study, which are in agrément with the findings of those authors, showed that variations generally occur in the accuracy of torque values of brackets when compared with the values recommended by the technique and different manufacturers. This finding represents another aspect that interferes with the final buccolingual position of teeth.

Torque is related to the dental position desired at the end of treatment, and it must be individualized for the achievement of correct intercuspation, esthetics, and adequate function ${ }^{14,16}$.

A change in the torque of brackets may have significant clinical consequences. This variability may cause either an end-to-end ratio or, more severely, an anterior crossbite when the inclination of the maxillary canine decreases or the mandibular one increases. Clinically, this result would be undesirable especially in patients with Class III malocclusion.

On the other hand, a torque increase in the maxillary canine bracket, or a reduction in the mandibular one, causes an increase in the overjet, hindering the treatment of patients with Class II malocclusion.

Torque is influenced by factors such as: anatomy of the buccal aspect of teeth, teeth size, bracket positioning, bracket size $^{7}$, gap between the wire and the bracket slot ${ }^{4,8-9}$, differences in direct bonding ${ }^{10}$, unusual crown shape and angulation ${ }^{11}$, application of strength outside the tooth center of resistance ${ }^{6}$, teeth with unusual morphology, and position of dental eruption ${ }^{14}$. The clinical responses for torque angle variations of brackets presented in this study should be interpreted carefully. Considering the nature of a laboratory study, it is very difficult to define precisely which changes in dental positions would occur due to the small variations in the accuracy of the torque of brackets, in light of the high number of variables associated with the final tooth position. Professionals should exercise attention to identify variables and controlling torques individually, especially in the final stages of the treatment.

Archambault et al. ${ }^{4}$ reported the existence of a gap between the wire and the slot of the bracket, which allows a torque variation. The authors observed that a gap in the wire with greatest diameter - 0.018 " x $0.025^{\prime \prime}$ - in a 0.018 " slot, and a $0.021 " \times 0.025^{\prime \prime}$ wire in a $0.22 "$ slot-created gaps of $2^{\circ}$ 
and $6^{\circ}$, respectively. The difference found between the torque angle values of brackets used in this study and the Roth prescription is lower than the gap of the last wire of the technique. Furthermore, the orthodontists often use a $0.019 " \times 0.025 "$ wire in a 0.022 " slot. This leads to an even larger gap of around $10^{\circ}$.

It is not possible to affirm that the statistical differences found between the brackets from some brands and the recommended norms could lead to clinically relevant outcomes. Further clinical studies are required to investigate the clinical outcomes of the discrepancies found between torque angles of brackets and their norms.

Considering the limitations of this study, it may be concluded that the medium torque angle values were significantly different for maxillary canine brackets from brands Abzil, Morelli and Ormco; for mandibular canines from brands Abzil, Eurodonto and Ormco regarding. Comparisons among the brands revealed significant differences for all brands, except for Morelli and Eurodonto, and Morelli and Ormco for maxillary canines. For mandibular canines, only the brands Abzil and Eurodonto differed significantly between each other.

Despite the differences found among the torque angles of the evaluated brackets, compared with those recommended in the prescription, these variations are clinically acceptable.

\section{References}

1. Andrews LF. Straight wire - the concept and the appliance. San Diego: Wells; 1989. 407p.

2. Moesi B, Dyer F, Benson PE. Roth versus MBT: does bracket prescription have an effect on the subjective outcome of pre-adjusted edgewise treatment? Eur J Orthod. 2011; 36: 1-8.

3. Jain M, Varghese J, Mascarenhas R, Mogra S, Shetty S, Dhakar N. Assessment of clinical outcomes of Roth and MBT bracket prescription using the American Board of Orthodontics Objective Grading System. Contemp Clin Dent. 2013; 4: 307-12.

4. Archambault A, Lacoursiere R, Badawi H, Major PW, Carey J, Flores Mir C. Torque expression in stainless steel orthodontic brackets. Angle Orthod. 2010; 80: 201-10.

5. Roth RH. The Straight-wire appliance 17 years later. J Clin Orthod. 1987; 21: $632-42$

6. Tominaga J, Chiang PC, Ozaki H, Tanaka M, Koga Y, Bourauel C, et al. Effect of play between bracket and archwire on anterior tooth movement in sliding mechanics: Athree-dimensional finite element study. J Dent Biomech. 2012; 3: 1-7.

7. Dolci GS, Spohr AN, Zimmer ER, Marchioro EE. Assessment of the dimensions and surface characteristics of orthodontic wires and bracket slots. Dental Press J Orthod. 2013; 18: 69-75.

8. Meireles JKS, Ursi W. Optimization of orthodontic treatment using the Centrex System to retract anterior teeth. Dental Press J Orthod. 2012; 17 : 29-44.

9. Arreghini A, Lombardo L, Mollica F, Siciliani G. Torque Expression capacity of 0.0018 and 0.022 bracket slots by changing archwire material and crosssection. Prog Orthod. 2014; 15: 61-79.

10. Ousehal L, Lazrak L. The accuracy of brackets placement in direct bonding technique: a comparison between the pole-like bracket positioning gauge and the star-like bracket positioning gauge. Open Journal of Stomatology. 2011; 1: 121-5.
11. Polak PT, Moro A, Bié MDD, Lopes SK, Spada PP, Moresca R, et al Influence of variation in the bracket positioning on the lingual surface of upper canines on the torque expression. Ortho Sci Orthod Sci Pract. 2010; 3: 315-9.

12. SardarianA, Danaei SM, Shahidi S, Boushehri SG, Geramy A. The effect of vertical bracket positioning on torque and the resultant stress in the periodontal ligament - a finite element study. Prog Orthod. 2014; 15: 50-60.

13. Houston WJB. The analysis of errors in orthodontic measurements. Am J Orthod. 1983; 83: 382-90.

14. Streva AM, Cotrim-Ferreira FA, Garib DG, Carvalho PEG. Are torque values of preadjusted brackets precise? J Appl Oral Sci. 2011; 19: 313-7.

15. Bóbbo MF. Torque evaluation to incisors brackets of the MBT technique. [Master's thesis]. São Paulo: Universidade Cidade de São Paulo; 2006. 108p.

16. Capistrano A, Cordeiro A, Siqueira DF, Capelozza Filho L, Cardoso MA, Almeida-Petrin RR. From conventional to self-ligant bracket systems: Is it possible to aggregate the experience with the former to the use of the latter? Dental Press J Orthod. 2014; 19: 139-57. 